\title{
Comparison of 3.0- and 1.5-T Three-dimensional Time-of-Flight MR Angiography in Moyamoya Disease: Preliminary Experience
}

\section{AUTHOR(S):}

Fushimi, Yasutaka; Miki, Yukio; Kikuta, Ken-ichiro; Okada, Tsutomu; Kanagaki, Mitsunori; Yamamoto, Akira; Nozaki, Kazuhiko; ... Hanakawa, Takashi; Fukuyama, Hidenao; Togashi, Kaori

\section{CITATION:}

Fushimi, Yasutaka ...[et al]. Comparison of 3.0- and 1.5-T Three-dimensional Time-ofFlight MR Angiography in Moyamoya Disease: Preliminary Experience. Radiology 2006, 239(1): 232-237

\section{ISSUE DATE:}

2006-04

URL:

http://hdl.handle.net/2433/218968

RIGHT:

(c) RSNA, 2006 
Comparison of 3.0- and 1.5-T Three-dimensional Time-ofFlight MR Angiography in Moyamoya Disease: Preliminary Experience $^{1}$

Yasutaka Fushimi, MD

Yukio Miki, MD, PhD

Ken-ichiro Kikuta, MD, PhD

Tsutomu Okada, MD

Mitsunori Kanagaki, MD, PhD

Akira Yamamoto, MD

Kazuhiko Nozaki, MD, PhD

Nobuo Hashimoto, MD, PhD

Takashi Hanakawa, MD, PhD

Hidenao Fukuyama, MD, PhD

Kaori Togashi, MD, PhD
${ }^{1}$ From the Department of Diagnostic Imaging and Nuclear Medicine (Y.F., Y.M., T.O., M.K., A.Y., K.T.), Department of Neurosurgery (K.K., K.N., N.H.), and Human Brain Research Center (T.H., H.F.), Kyoto University Graduate School of Medicine, 54 Shogoin-Kawaharacho, Sakyo-ku, Kyoto, 606-8507, Japan. From the 2005 RSNA Annual Meeting. Received November 29, 2004; revision requested January 20, 2005; revision received April 3; final version accepted May 2. Supported in part by a grant from the Ministry of Education Culture, Sports, Science and Technology, Japan. Address correspondence to Y.M. (e-mail: mikiy@kuhp.kyoto-u.ac.jp).
Purpose:

Materials and Methods:

Conclusion:
To prospectively compare 3.0 - and 1.5 - $\mathrm{T}$ three-dimensional (3D) time-of-flight (TOF) magnetic resonance (MR) angiography in patients with moyamoya disease, with special emphasis on the visualization of abnormal netlike vessels (moyamoya vessels).

Study protocols were approved by the local ethics committee; written informed consent was obtained from all patients. The study included 24 consecutive patients with moyamoya disease (four male and 20 female patients). Patients ranged in age from 17 to 66 years (mean age, 41 years). Moyamoya disease had been diagnosed in all patients before they were entered into the study. All patients underwent 3D TOF MR angiography at both 3.0 and $1.5 \mathrm{~T}$; imaging examinations were performed within 14 days of each other. Maximum intensity projections (MIPs) obtained with MR angiography performed at both 3.0 and 1.5 $\mathrm{T}$ were evaluated by two neuroradiologists; the visualization of moyamoya vessels was graded according to a 4-point scale. For both 3.0- and 1.5-T imaging, the number of high-signal-intensity areas and the summation of crosssectional areas of high signal intensity on source images obtained at the same level of MR angiography were compared quantitatively by using the Wilcoxon matched-pair signed-rank test.

Results: Moyamoya vessels were better visualized on MIPs obtained with 3.0-T imaging than on MIPs obtained with 1.5-T imaging $(P<.001)$. At the identical level of the source image, 3.0-T imaging depicted more high-signalintensity areas than did 1.5-T imaging. Wider cross-sectional areas of moyamoya vessels were visualized with 3.0-T imaging than with 1.5 - $\mathrm{T}$ imaging $(P<.001)$.

Moyamoya vessels are better depicted with MR angiography at $3.0 \mathrm{~T}$ than at $1.5 \mathrm{~T}$.

๑ RSNA, 2006 
M oyamoya disease is a rare cerebrovascular occlusive disease of unknown origin and is characterized by stenosis or occlusion of both internal carotid arteries (ICAs) at the supraclinoid portion $(1,2)$. Although this disease occurs predominantly in Asian populations, particularly in $\mathrm{Ja}^{-}$ pan, it has also been reported in other countries (3-5). The major symptoms of moyamoya disease are related to age, with transient ischemic attack often seen in pediatric patients and intracranial hemorrhage often seen in adults.

The principal angiographic feature of moyamoya disease is bilateral stenosis or occlusion of the supraclinoid portions of the ICAs, potentially extending to the

\begin{tabular}{|c|c|c|}
\hline \multicolumn{3}{|c|}{$\begin{array}{l}\text { Scores in the Depiction of Moyamoya } \\
\text { Vessels }\end{array}$} \\
\hline $\begin{array}{l}\text { Patient } \\
\text { No. }\end{array}$ & $\begin{array}{l}1.5-\mathrm{T} \\
\text { Field Strength }\end{array}$ & $\begin{array}{l}\text { 3.0-T } \\
\text { Field Strength }\end{array}$ \\
\hline 1 & 2 & 3 \\
\hline 2 & 2 & 3 \\
\hline 3 & 1 & 2 \\
\hline 4 & 2 & 3 \\
\hline 5 & 2 & 2 \\
\hline 6 & 2 & 2 \\
\hline 7 & 3 & 3 \\
\hline 8 & 1 & 2 \\
\hline 9 & 2 & 3 \\
\hline 10 & 2 & 2 \\
\hline 11 & 3 & 3 \\
\hline 12 & 3 & 3 \\
\hline 13 & 2 & 3 \\
\hline 14 & 2 & 3 \\
\hline 15 & 2 & 3 \\
\hline 16 & 1 & 3 \\
\hline 17 & 2 & 3 \\
\hline 18 & 2 & 3 \\
\hline 19 & 2 & 3 \\
\hline 20 & 1 & 3 \\
\hline 21 & 1 & 3 \\
\hline 22 & 2 & 3 \\
\hline 23 & 3 & 3 \\
\hline 24 & 1 & 3 \\
\hline
\end{tabular}

Note.-Scores used in the depiction of moyamoya vessels are as follows: $3=$ excellent (vessel segments were clearly and continuously visualized, and vesseltissue contrast is high), 2 = visible (vessel segments are visualized and adequate for a confident diagnosis, but vessel-tissue contrast is not particularly high), $1=$ scarcely visible (vessel segments were visualized but inadequate for diagnosis), and $0=$ not visible. proximal portions of the anterior cerebral arteries and middle cerebral arteries (MCAs), with the presence of abnormal netlike vessels (moyamoya vessels) in the basal areas (1). Leptomeningeal collateral vessels from the posterior cerebral arteries or transdural collateral vessels from the external carotid arteries may also be present (1).

With the development of magnetic resonance (MR) imaging techniques, the diagnosis of moyamoya disease with MR imaging and MR angiography has become possible (6). When ICA occlusion and moyamoya vessels are demonstrated at MR angiography, conventional angiography is unnecessary, particularly in pediatric patients (6). Interpretation of highquality images is crucial when diagnosing moyamoya disease with MR imaging without conventional angiography. Precise evaluation of abnormal vascular networks in the basal ganglia, in addition to vascular occlusion, is essential for securing a definitive diagnosis of moyamoya disease (6-8).

MR imaging techniques with 3.0-T imaging have gradually become prevalent. Imaging at 3.0 T provides a better signal-to-noise ratio, which increases approximately linearly with constant magnetic induction field from 1.5 - to 3.0-T imaging $(9,10)$. The $\mathrm{T} 1$ relaxation time increases at higher magnetic field strengths, and this produces improved vessel-tissue contrast at 3.0-T imaging (11-13). A better signal-to-noise ratio and increased $\mathrm{T} 1$ relaxation time at 3.0-T imaging contribute to the improved quality of MR angiography. Furthermore, Willinek et al (14) demonstrated that, in the diagnosis of cerebrovascular occlusive disease, high-spatial-resolution three-dimensional (3D) time-of-flight (TOF) MR angiography at $3.0 \mathrm{~T}$ is superior to that at $1.5 \mathrm{~T}$. To our knowledge, however, no reports have focused on the comparison of 3.0- and 1.5-T MR angiography in patients with moyamoya disease.

Thus, the purpose of our study was to prospectively compare 3.0 - and 1.5 - T 3D TOF MR angiography in patients with moyamoya disease, with special emphasis on the visualization of moyamoya vessels.

\section{Materials and Methods}

\section{Study Design and Patients}

A prospective study was performed with 24 consecutive patients with moyamoya disease (four male and 20 female patients) between December 2003 and September 2004. Moyamoya disease had been diagnosed in all patients with conventional angiography before they were entered into our study (6). The mean patient age was 41 years (age range, $17-66$ years). Patients were initially suspected of having moyamoya disease due to transient ischemic attack or cerebral infarction $(n=14)$, intracranial hemorrhage $(n=9)$, or incidental findings at MR imaging after a traffic accident $(n=1)$. Of the 24 patients, seven $(29 \%)$ had not undergone any surgical synangiosis procedure. Seventeen of the 24 patients (71\%) had undergone creation of a superficial temporal artery (STA)-MCA anastomosis combined with encephalomyosynangiosis $(n=15)$ or encephaloduroarteriosynangiosis $(n=2)$. All patients had undergone regular follow-up. In these 17 patients, the mean postoperative duration at the time of our study was 89 months (range, 7-288 months).

Study protocols were approved by the local ethics committee, and all pa-

\section{Published online before print 10.1148/radiol.2383042020 \\ Radiology 2006; 239:232-237 \\ Abbreviations: \\ ICA = internal cerebral artery \\ $\mathrm{MCA}=$ middle cerebral artery \\ MIP = maximum intensity projection \\ STA = superficial temporal artery \\ $3 \mathrm{D}=$ three dimensional \\ TOF $=$ time of flight}

\section{Author contributions:}

Guarantors of integrity of entire study, all authors; study concepts/study design or data acquisition or data analysis/interpretation, all authors; manuscript drafting or manuscript revision for important intellectual content, all authors; approval of final version of submitted manuscript, all authors; literature research, Y.M., K.K., M.K. A.Y., K.N., T.H., H.F.; clinical studies, Y.F., K.K., T.O., K.N., T.H., H.F.; statistical analysis, Y.F., Y.M., M.K., A.Y K.N., N.H., H.F., K.T.; and manuscript editing, Y.F., Y.M., T.O., N.H., H.F., K.T.

Authors stated no financial relationship to disclose. 
tients provided written informed consent before entering the study. When the patient was a minor, additional written informed consent was also obtained from the parent.

\section{MR Imaging}

Our study was performed with 3.0-T (Magnetom Trio; Siemens, Erlangen, Germany) and 1.5-T (Magnetom Symphony; Siemens) MR units. Circular polarized head coil arrays were used for both MR units. The following parameters were used for 3.0-T MR angiography: 22.0/3.7 (repetition time msec/ echo time msec), $20^{\circ}$ flip angle, 0.8mm-thick sections, $200 \times 200$-mm field of view with a $512 \times 208$ matrix, effective voxel size of $0.39 \times 0.96 \times 0.8 \mathrm{~mm}$, and acquisition time of 5 minutes 51 seconds. The following parameters were used for 1.5-T MR angiography: $35 / 7$, 0.8-mm-thick sections, $20^{\circ}$ flip angle, $200 \times 200$-mm field of view with a $512 \times 208$ matrix, effective voxel size of $0.39 \times 0.96 \times 0.8 \mathrm{~mm}$, and acquisition time of 6 minutes 21 seconds. The repetition time and echo time could not be matched because of the limitations of specific absorption rate with the 3.0-T MR system.

\section{Image Analysis}

Transverse and lateral maximum intensity projections (MIPs) obtained with 3.0- and 1.5-T MR angiography were independently assessed by two neuroradiologists (Y.F. and Y.M., with 8 and 19 years of experience, respectively). Lateral MIPs were reconstructed with data from the ipsilateral hemisphere. To evaluate the visibility of moyamoya vessels under identical circumstances, we evaluated only transverse and bilateral MIP images instead of reviewing all the angles of MIP images. The visibility of moyamoya vessels was assessed and scored as follows: 3, excellent (vessel segments were clearly and continuously visualized, and vessel-tissue contrast appears to be high); 2, visible (vessel segments are visualized and adequate for a confident diagnosis, but vessel-tissue contrast does not appear to be particularly high); 1 , scarcely visible (vessel segments were visualized but inade-

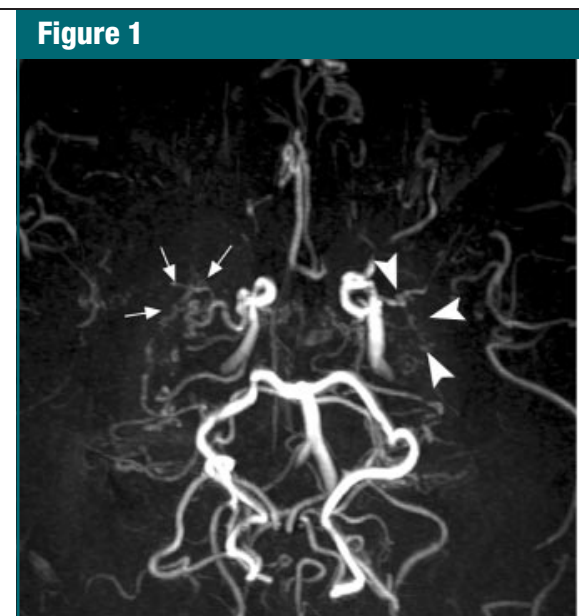

a.

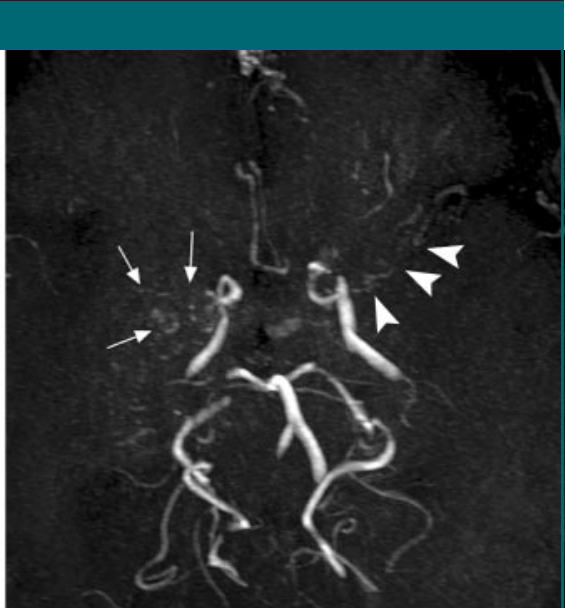

d.

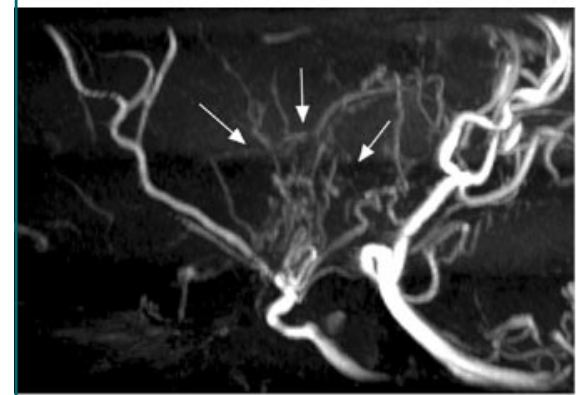

b.

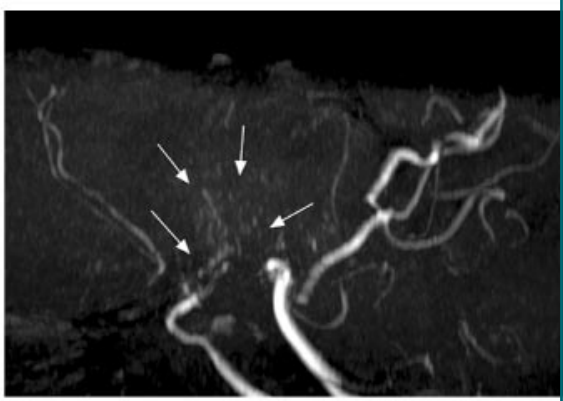

e.

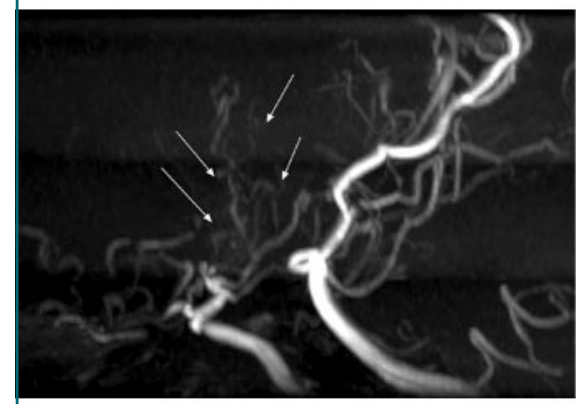

c.

Figure 1: MIPs from 3D TOF MR angiography in a 58-year-old woman show moyamoya vessels (arrows) from ICAs. (a) Transverse MIP, (b) lateral MIP of the right side, and (c) lateral MIP of the left side from 3.0-T MR angiography (22.0/3.7). (d) Transverse MIP, (e) lateral MIP of the right side, and (f) lateral MIP of the left side from 1.5-T MR angiography (35/7). Transverse MIPs reveal more moyamoya vessels on the right side from the right ICA than on the left side. Moyamoya vessels from the right ICA are more clearly seen with 3.0-T imaging than with 1.5-T imaging. On the left side, 1.5-T MR angiography shows slight and discontinuous moyamoya vessels (arrowheads in d), whereas 3.0-T MR angiography shows fine moyamoya vessels (arrowheads in a) continuously and more clearly. MIPs from 3.0-T imaging were scored as 3 (excellent) and those from 1.5-T imaging were scored as 2 (visible).

quate for diagnosis); and 0 , not visible. Readers were blinded to field strength; discrepancies between the two readers were resolved by consensus. A consensus reading was required in one patient for 3.0 - $\mathrm{T}$ images $(4.2 \%)$ and in three patients for 1.5-T images $(12.5 \%)$.

A particle-counting method was used for source images from MR angiography. With use of ImageJ software, a 
Java-based image analysis program developed at the U.S. National Institutes of Health (http://rsb.info.nih.gov/ij /index.html), high signal intensity in the basal ganglia of the anterior circulation, which correspond to moyamoya vessels (1), were manually selected and semiautomatically counted by one neuroradiologist (Y.F.). Image selection from 3.0- and 1.5-T MR angiographic data obtained in the same patient was performed simultaneously, as identical section levels can be selected; this enabled comparison of images obtained at 3.0 and $1.5 \mathrm{~T}$. Image selection procedures were performed with two personal computers by using ExaVision Lite soft-

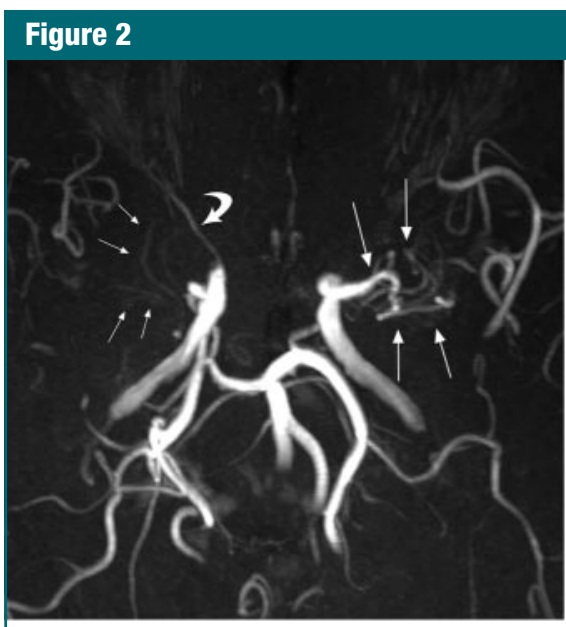

a.

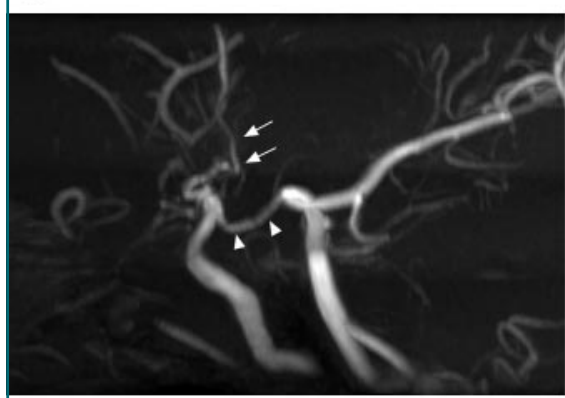

b.

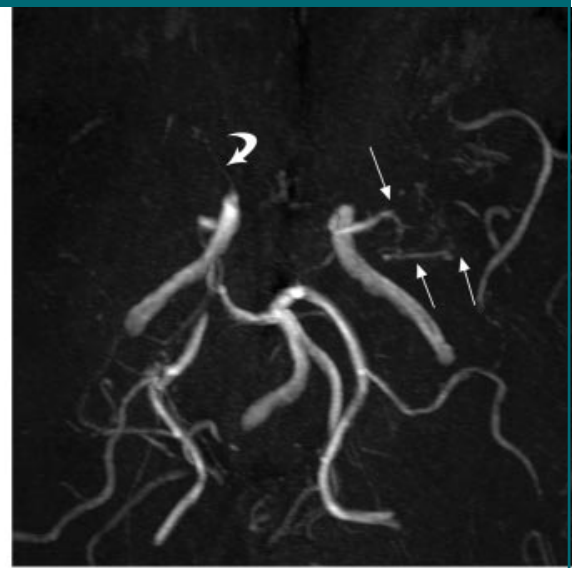

c.

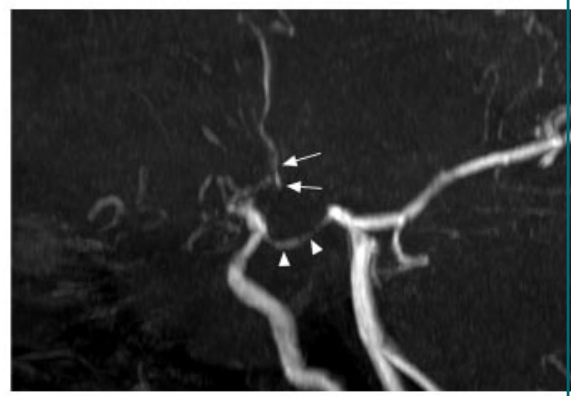

Figure 2: $\quad$ MIPs from 3D TOF MR angiography in a 49-year-old man. (a) Transverse MIP and (b) lateral MIP of the left side from MR angiography (22.0/3.7). (c) Transverse MIP and (d) lateral MIP of the left side from 1.5-T MR angiography (35/7). Images obtained at 3.0 T reveal prominent moyamoya vessels on the left side (long straight arrows in $\mathbf{a}$, arrows in $\mathbf{b}$ ) and scarce moyamoya vessels on the right side (short straight arrows in a). Images obtained at $1.5 \mathrm{~T}$ show moyamoya vessels on the left side (straight arrows in c, arrows in d) but no apparent moyamoya vessels on the right side. Note that the posterior communicating artery (arrowheads in $\mathbf{b}$ and $\mathbf{d}$ ) and right ophthalmic artery (curved arrow in $\mathbf{a}$ and $\mathbf{c}$ ) are visualized more clearly at $3.0 \mathrm{~T}$ than at 1.5 T. MIPs from 3.0-T imaging were scored as 3 (excellent), whereas images obtained at 1.5 T were scored as 2 (visible) ware (Ziosoft, Tokyo, Japan). Regions of interest were carefully selected in biateral basal ganglia without including cisternal structures because major spectively. We determined the threshold for the source images with MR anbrain parenchyma just black out to minmize the variances of the thresholds and obtained the binary data from MR angiography. The number of high signal sectional areas of high signal intensity, which correspond to the cross-sectional and spheric fissure and sylvian fissure, regiography to make the brightest part of area of moyamoya vessels, were obtained for 3.0- and 1.5-T imaging.

\section{Statistical Analysis}

For statistical evaluation, scores for MIPs obtained with MR angiography, the number of high-signal-intensity areas, and the summation of cross-sectional areas of high signal intensity were compared by using the Wilcoxon matched-pair signed-rank test with software (JMP 5.0; SAS Institute, Cary, $\mathrm{NC}$ ). This was done because the Shapiro-Wilk test was performed for normality and the null hypothesis rejected. A $P$ value of less than .05 was considered to indicate a statistically significant difference.

\section{Results}

MIPs were obtained with 3D TOF MR angiography in the 24 patients (Table) (Figs 1, 2). MIPs from 3.0-T MR angiography depicted moyamoya vessels more clearly than did those from 1.5-T MR angiography $(P<.001)$.

The number of high-signal-intensity areas in anterior circulations (anterior cerebral artery and MCA territories) on source images obtained at both 3.0 and 1.5 $\mathrm{T}$ showed that the number of highsignal-intensity areas at 3.0-T imaging was greater than that at $1.5-\mathrm{T}$ imaging $(P<.001)$ (Figs 3, 4). Cross-sectional areas of high-signal-intensity were larger at 3.0-T imaging than at $1.5-\mathrm{T}$ imaging $(P<.001)$ (Fig 5).

\section{Discussion}

Our results demonstrate that moyamoya vessels are better depicted with MIPs from 3.0-T MR angiography than with those from 1.5-T MR angiography. More moyamoya vessels (in both number and cross-sectional area) were detected at 3.0-T imaging than at 1.5-T imaging. The particle-counting method with use of ImageJ software has been applied to cell counting in biologic studies $(15,16)$. In our study, we used this method in the postprocessing of MR angiographic data to assess moyamoya vessels. This method may be useful for objective and longitudinal evaluations 


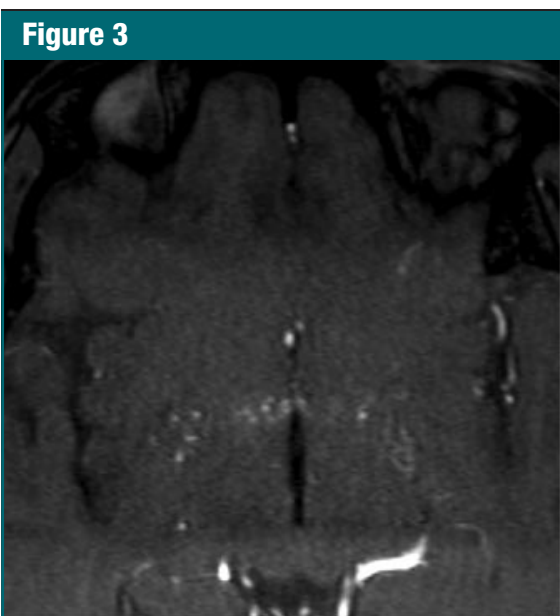

a.

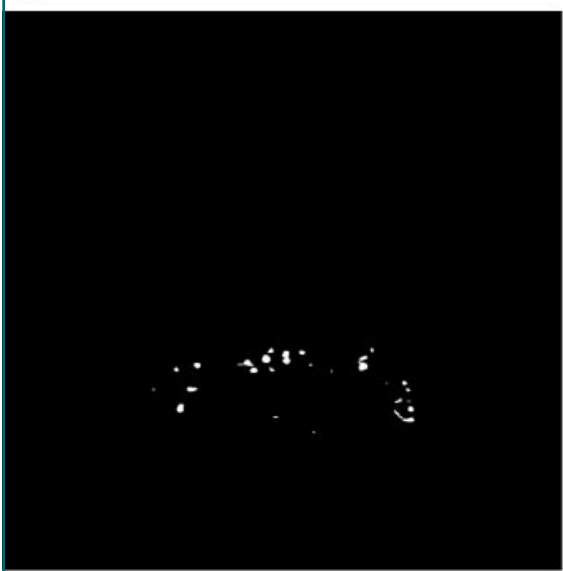

b.

d.

Figure 3: Images obtained in a 58-year-old woman. Source images from MR angiography obtained at the same level at (a) $3.0 \mathrm{~T}$ (22.0/3.7) and (c) $1.5 \mathrm{~T}$ (35/7). More high-signal-intensity areas in both basal ganglia were seen at 3.0-T imaging than at 1.5-T imaging. The area of anterior circulation was selected and changed into binary data. The number of high-signal-intensity areas was calculated semiautomatically, and the total number of cross-sectional areas of high signal intensity was calculated by using software. (b) Binary data image obtained at 3.0 T shows more high-signal-intensity areas than (d) data from the source image obtained at $1.5 \mathrm{~T}$.

because the number and cross-sectional area of moyamoya vessels are provided.

Moyamoya disease is a rare cerebrovascular disease. In adult patients, intracerebral hemorrhage is one of the most common complications at disease onset (17-20). For patients with moyamoya disease and hemorrhage, repeat bleeding is the most crucial event that affects quality of life $(21,22)$. The mechanisms of repeat bleeding supposedly involve rupture of moyamoya vessels. Moyamoya vessels have a tendency to contract; when that happens, the re-

c.
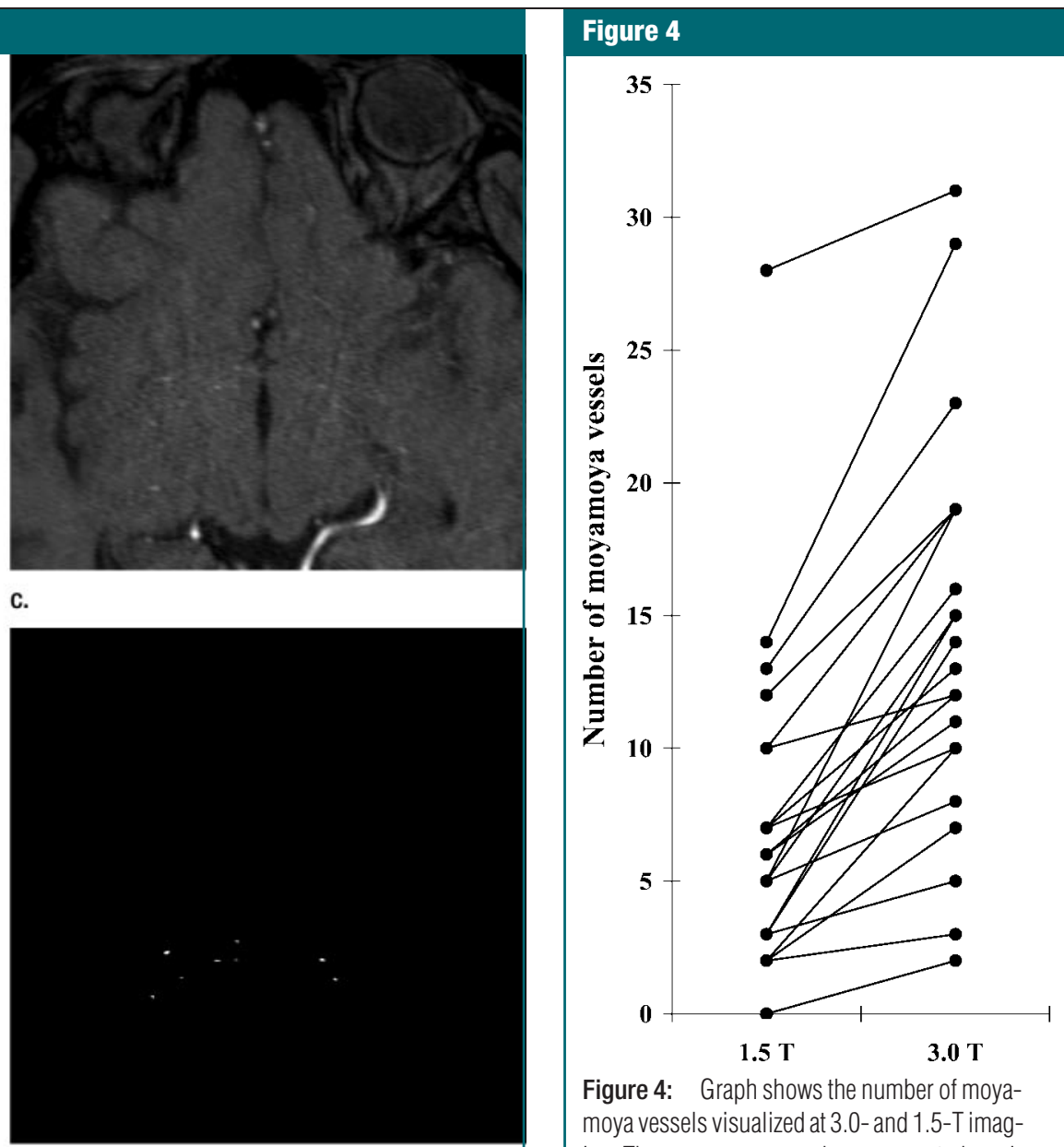

Figure 4: Graph shows the number of moyamoya vessels visualized at 3.0- and 1.5-T imaging. The moyamoya vessels were counted semiautomatically on MR angiography source images obtained at the same levels. More high-signalintensity areas were seen at $3.0 \mathrm{~T}$ than at $1.5 \mathrm{~T}$.

study was a preliminary study that targeted moyamoya disease. To our knowledge, this is the first study in which the visibility of moyamoya vessels has been described at both 3.0- and 1.5-T MR angiography.

One limitation of our study is that most of our patients (71\%) had undergone surgical treatment. After bypass surgery (creation of STA-MCA anastomosis, encephalomyosynangiosis, and encephaloduroarteriosynangiosis), collateral arteries develop from the external cerebral artery and the amount of moyamoya vessels decreases in about $50 \%$ of patients (8). In our study, only seven patients had not undergone surgery; thus, a more precise interpretation of our results would be to say that 


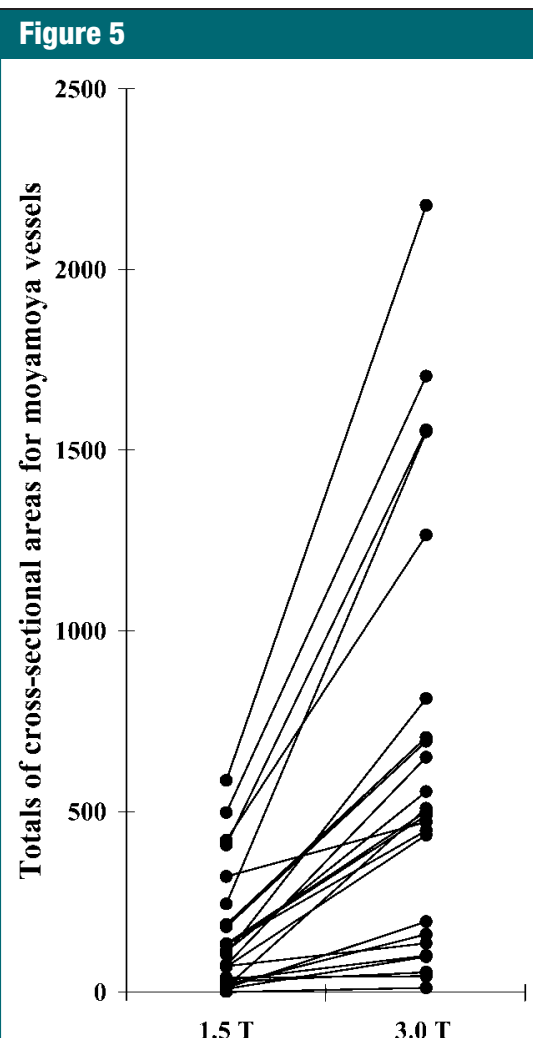

Figure 5: Graph shows the cross-sectional area of moyamoya vessels. The total cross-sectional areas for moyamoya vessels at the same level of MR angiography source images were calculated semiautomatically and compared between 3.0- and 1.5-T MR systems. Larger cross-sectional areas were visualized with 3.0-T imaging than with 1.5-T imaging. The total cross-sectional area is given as pixels.

our study demonstrated the advantages of 3.0-T imaging over 1.5-T imaging for follow-up evaluation. Although 3.0-T imaging may indeed prove advantageous in the diagnosis of moyamoya disease, larger numbers of preoperative patients should be studied in the future. In preoperative treatment planning, conventional angiography would be required because it can provide more detailed information about moyamoya vessels and other major vessels than does 3.0-T MR angiography; thus, comparative studies between 3.0-T MR angiography and conventional angiography may be needed in the future. We did not evaluate stenosis or dilatation of ICAs, anterior cerebral arteries, and MCAs because most patients had undergone conventional angiography several years before the MR examinations, which made it difficult for us to verify the findings with use of a reference standard. This is another limitation. In our study, visualization of STA-MCA bypass was not evaluated because aliasing artifacts affected STA-MCA bypass in some patients, STA-MCA bypass sites were not included in the fields of view in all patients, and most patients had undergone postoperative angiography several years before the MR examinations. Further studies may be needed to evaluate the findings of MR angiography in cases of STA-MCA bypass. Although the readers were blinded to the field strength of MR angiographic images, there were some differences in image quality of moyamoya vessels between 3.0- and 1.5-T images. It is possible that the readers were influenced by these differences.

In conclusion, moyamoya vessels are better depicted at 3.0-T 3D TOF MR angiography than at $1.5-\mathrm{T} 3 \mathrm{D}$ TOF MR angiography. Radiologists must be aware of the differences, especially when patients undergo follow-up MR angiography with both 3.0- and 1.5-T MR systems.

\section{References}

1. Suzuki J, Takaku A. Cerebrovascular "moyamoya" disease: disease showing abnormal net-like vessels in base of brain. Arch Neurol 1969;20: $288-299$

2. Suzuki J, Kodama N. Moyamoya disease: a review. Stroke 1983;14:104-109.

3. Fukui M. Current state of study on moyamoya disease in Japan. Surg Neurol 1997;47:138-143.

4. Chiu D, Shedden P, Bratina P, Grotta JC. Clinical features of moyamoya disease in the United States. Stroke 1998;29:1347-1351.

5. Graham JF, Matoba A. A survey of moyamoya disease in Hawaii. Clin Neurol Neurosurg 1997; 99(suppl 2):S31-S35.

6. Fukui M. Guidelines for the diagnosis and treatment of spontaneous occlusion of the circle of Willis ('moyamoya' disease). Research Committee on Spontaneous Occlusion of the Circle of Willis (Moyamoya Disease) of the Ministry of Health and Welfare, Japan. Clin Neurol Neurosurg 1997;99(suppl 2):S238-S240.

7. Fujisawa I, Asato R, Nishimura K, et al. Moyamoya disease: MR imaging. Radiology 1987;164: 103-105.

8. Yoon HK, Shin HJ, Lee M, Byun HS, Na DG, Han BK. MR angiography of moyamoya disease before and after encephaloduroarteriosynangiosis. AJR Am J Roentgenol 2000;174:195-200.

9. Bernstein MA, Huston J III, Lin C, Gibbs GF,
Felmlee JP. High-resolution intracranial and cervical MRA at 3.0T: technical considerations and initial experience. Magn Reson Med 2001;46: 955-962.

10. Edelstein WA, Glover GH, Hardy CJ, Redington RW. The intrinsic signal-to-noise ratio in NMR imaging. Magn Reson Med 1986;3:604-618.

11. Campeau NG, Huston J III, Bernstein MA, Lin C, Gibbs GF. Magnetic resonance angiography at 3.0 Tesla: initial clinical experience. Top Magn Reson Imaging 2001;12:183-204.

12. Al-Kwifi O, Emery DJ, Wilman AH. Vessel contrast at three Tesla in time-of-flight magnetic res onance angiography of the intracranial and carotid arteries. Magn Reson Imaging 2002;20:181187.

13. Thomas SD, Al-Kwifi O, Emery DJ, Wilman AH Application of magnetization transfer at $3.0 \mathrm{~T}$ in three-dimensional time-of-flight magnetic resonance angiography of the intracranial arteries. J Magn Reson Imaging 2002;15:479-483.

14. Willinek WA, Born M, Simon B, et al. Time-of flight MR angiography: comparison of 3.0-T imag ing and 1.5-T imaging-initial experience. Radiology $2003 ; 229: 913-920$.

15. Gering E, Atkinson CT. A rapid method for count ing nucleated erythrocytes on stained blood smears by digital image analysis. J Parasitol 2004; 90:879-881.

16. Rajwa B, McNally HA, Varadharajan P, Sturgis J, Robinson JP. AFM/CLSM data visualization and comparison using an open-source toolkit. Microse Res Tech 2004;64:176-184.

17. Morioka M, Hamada J, Todaka T, Yano S, Kai Y, Ushio Y. High-risk age for rebleeding in patients with hemorrhagic moyamoya disease: long-term follow-up study. Neurosurgery 2003;52:10491054.

18. Kobayashi E, Saeki N, Oishi H, Hirai S, Yamaura A. Long-term natural history of hemorrhagic moyamoya disease in 42 patients. J Neurosurg 2000;93:976-980.

19. Okada $\mathrm{Y}$, Shima $\mathrm{T}$, Nishida $\mathrm{M}$, Yamane $\mathrm{K}$, Yamada T, Yamanaka C. Effectiveness of superfi cial temporal artery-middle cerebral artery anas tomosis in adult moyamoya disease: cerebral he modynamics and clinical course in ischemic and hemorrhagic varieties. Stroke 1998;29:625-630.

20. Kikuta K, Takagi Y, Nozaki K, et al. Asymptomatic microbleeds in moyamoya disease: $\mathrm{T} 2{ }^{*}$-weighted gradient-echo magnetic resonance imaging study. J Neurosurg 2005; 102:470-475.

21. Yamashita M, Oka K, Tanaka K. Histopathology of the brain vascular network in moyamoya disease. Stroke 1983;14:50-58.

22. Morioka M, Hamada J, Kawano T, et al. Angiographic dilatation and branch extension of the anterior choroidal and posterior communicating arteries are predictors of hemorrhage in adult moyamoya patients. Stroke 2003;34:90-95.

23. Kagawa R, Okada Y, Moritake K, Takamura M Magnetic resonance angiography demonstrating adult moyamoya disease progressing from unilateral to bilateral involvement: case report. Neurol Med Chir (Tokyo) 2004;44:183-186.

24. Kim SK, Seol HJ, Cho BK, Hwang YS, Lee DS, Wang KC. Moyamoya disease among young patients: its aggressive clinical course and the role of active surgical treatment. Neurosurgery 2004 $54: 840-844$. 